Proceedings of the American Control Conference

Philadelphia, Pennsylvania • June 1998

\title{
An Estimation-Based Approach to the Design of Adaptive IIR Filters ${ }^{1}$
}

\author{
Bijan Sayyarrodsari ${ }^{2}$
}

Jonathan P. How ${ }^{3}$
Alain Carrier ${ }^{5}$

\begin{abstract}
We present an estimation-based approach to the design of adaptive IIR filters. We also use this approach to design adaptive filters when a feedback signal from the output of the adaptive filter contaminates the reference signal. We use an $H_{\infty}$ criterion to cast the problem as a nonlinear $H_{\infty}$ filtering problem, and present an approximate linear $H_{\infty}$ filtering solution. This linear filtering solution is then used to adapt the adaptive IIR Filter. The presentation of the proposed adaptive algorithm is done in the context of an adaptive Active Noise Cancellation (ANC) problem. Simulations are used to examine the performance of the proposed estimation-based adaptive algorithm.
\end{abstract}

\section{Introduction}

The Least-Mean Squares (LMS) adaptive algorithm [1] has been used for over 35 years as the center piece of a wide variety of adaptive algorithms. Despite numerous successful applications, it was only recently that the $H_{\infty}$ optimality of the LMS algorithm was established [2], and its important properties, such as bounds on adaptation rates, were rigorously derived.

Over years, however, numerous (mostly heuristic) variations of the LMS algorithm have been developed to overcome practical implementation problems (see [1] and [4] for instance). The contamination of the reference signal (see Figure 1) with the output of the adaptive filter has proven to further complicate the implementation problems. Thus systematic approaches for the design and analysis of the adaptive filters for realistic control scenarios have been of primary interest to researchers in the field. In [3], an estimation-based approach to the design of adaptive FIR filters is proposed. Reference [3] uses an adaptive ANC scenario to explain how an estimation interpretation of the adaptive control problem provides a framework for the systematic synthesis and analysis of adaptive FIR filters. This paper extends the results in [3] to the design of adaptive Infinite Impulse Response (IIR) filters. The formulation presented here also applies when the reference signal is contaminated with a feedback from the output of the adaptive filter (Figure 1).

The effects of the reference signal contamination has been studied in the adaptive control literature (see Chapter 3 of [4] and the references therein). Feedback neutralization

\footnotetext{
${ }^{1}$ Supported by Lockheed Martin Missiles \& Space Grant.

${ }^{2}$ Doctoral Candidate, Department of Electrical Engineering, Stanford University, bsr@sun-valley.stanford.edu

${ }^{3}$ Assistant Professor, Department of Aeronautics and Astronautics, Stanford University, howjo@sun-valley.stanford.edu

${ }^{4}$ Department of Electrical Engineering, Stanford University, hassibi@isl.stanford.edu

${ }^{5}$ Research Scientist, Advanced Technology Center, Lockheed Martin Palo Alto, alain@control3.atc.Imms.Imco.com

$0-7803-4530-4 / 98 \$ 10.00$ (C) 1998 AACC
}

is one approach which uses a separate feedback within the adaptive controller to cancel the effect of the undesired feedback [6]. This scheme, however, requires special care in the implementation to avoid the cancellation of the reference signal all together (see [4] for details). Looking at feedback neutralization from a different point of view, it is clear that this algorithm generates poles as well as zeros for the overall adaptive filter. In other words, the overall adaptive controller is an IIR filter in general (and hence stability of the overall system should be closely monitored). Observing this fact, there have been several attempts to directly design an adaptive IIR filter in such circumstances. Filtered-U recursive LMS algorithm is one such approach. In this technique, the feedback path is explicitly treated as part of the plant, [7], and the derivation involves approximations that rely on slow adaptation. Furthermore, there are many concerns about the convergence properties of this scheme [4]. It is also noted that the optimal solution can be extremely ill-conditioned if a large number of controller coefficients are used or the structure of feedback path is complicated.

This paper's estimation-based approach to the design of adaptive IIR filters, in essence meets a disturbance attenuation criterion (to be defined shortly), and hence provides a framework in which the questions about convergence and stability of the adaptive algorithm can be systematically addressed. Moreover, the estimation-based approach easily extends to the case where the reference signal available to the adaptive algorithm is contaminated with the feedback from the output of the adaptive filter.

This paper is organized as follows. Section 2 presents the estimation-based formulation for the adaptive filter design. Section 3 discusses the $H_{\infty}$-optimal solution to the formulated estimation problem. Section 4 outlines our proposed implementation scheme for the adaptive algorithm. Section 5 contains simulation results. Section 6 concludes this paper with a summary and final remarks.

\section{Problem Formulation}

We discuss the estimation-based approach to the design of an adaptive IIR filter (with and without the presence of a feedback path) in the context of the ANC problem of Figure 1. In this section we first concentrate on the case where there is no feedback path. The case with feedback path is then an straightforward extension.

The objective of ANC is to generate a control signal $u(k)$ such that the output of the secondary path, $y(k)$, is in some measure (to be specified later) close enough to the output of the primary path, $d(k)$. For this to materialize, the series connection of the IIR filter (for some optimal setting of its parameters) and the known secondary path must appropriately approximate the unknown pri- 


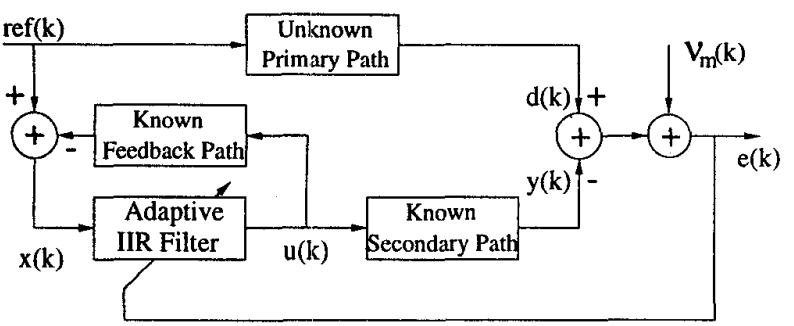

Fig. 1: Block Diagram of an ANC Problem With Feedback Contamination of the Reference Signal

mary path. Figure 2 captures this notion. This observation, in our view, is an estimation interpretation of the adaptive control problem. Note that as long as the output of the "modeling error" block in Figure 2 is bounded, then it can be treated as a component of the measurement disturbance signal, $\mathcal{V}_{m}(k)$.

To formalize this observation, we assume a state space model for the IIR filter and the secondary path, and then form an approximate model for the primary path as shown in Figure 3. This approximate model is the closest to the actual primary path for some optimal (but unknown) setting of the filter parameters. Now, the estimation-based approach to the design of an adaptive IIR filter is described as follows:

1. Devise an estimation strategy that recursively improves our estimate of the optimal values of the IIR filter parameters in the approximate model of the primary path (given the available measurement history to be described shortly),

2. Set the actual value of the parameters in the adaptive IIR filter to the best estimate of the parameters obtained from the estimation strategy.

We now take a closer look at the main signals in Figure 2. Note that $e(k)=d(k)-y(k)+\mathcal{V}_{m}(k)$, where

a) $e(k)$ is the available error measurement,

b) $V_{m}(k)$ is the exogenous disturbance that captures measurement noise, modeling error and initial condition uncertainty for the secondary path,

c) $y(k)$ is a known signal, because (i) $u(k)$ is exactly known (we directly set the parameters in the IIR filter), and (ii) we assume that $\theta_{0}$ (the initial condition for the secondary path) is known ${ }^{1}$.

The derived measured quantity, $m(k)$ in Figure 3, can now be defined as

$$
m(k) \triangleq e(k)+y(k)=d(k)+\nu_{m}(k)
$$

\subsection{Estimation Problem}

Figure 3 reflects a block diagram representation of the approximate model to the primary path. We assume a

\footnotetext{
${ }^{1}$ Note that as long as the effect of the initial condition in the output of the secondary path does not grow without bound, any error in $y(k)$ (due to an initial condition other than what we assumed) can be treated as a component; of the measurement disturbance.
}

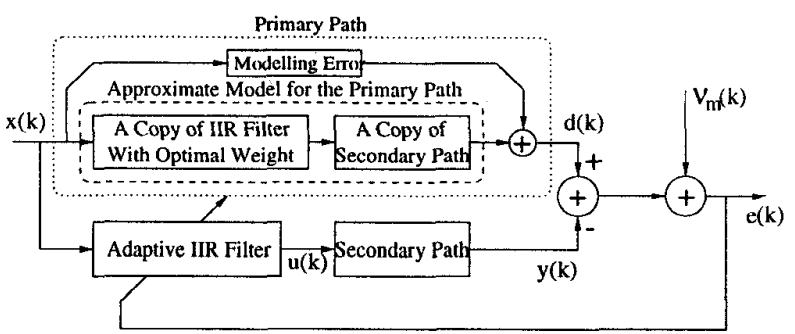

Fig. 2: Estimation Interpretation of the IIR Adaptive Filter Design in a ANC Problem

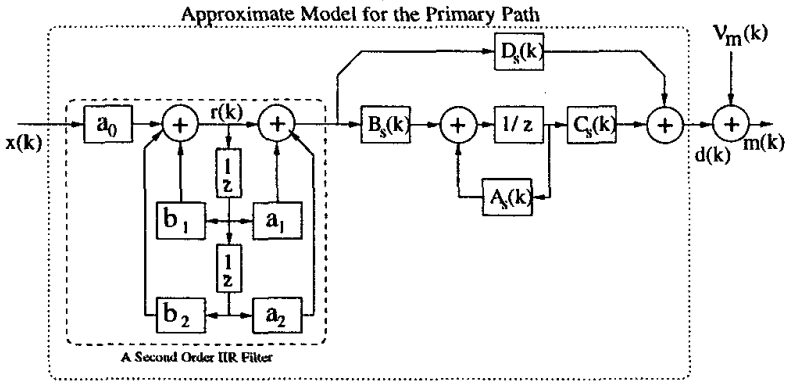

Fig. 3: Approximate Model For the Unknown Primary Path (A second order IIR filter is shown here)

state space model, $\left[A_{s}(k), B_{s}(k), C_{s}(k), D_{s}(k)\right]$, for the replica of the secondary path in Figure 3 . We also define $W(k)=\left[a_{0}(k) b_{1}(k) \cdots b_{N}(k) a_{1}(k) \cdots a_{N}(k)\right]^{T}$ to be the unknown optimal vector of the IIR filter parameters at time $k . \xi_{k}^{T}=\left[W(k)^{T} \theta(k)^{T}\right]$ is then the state vector for the overall system. Note that $\theta(k)$ captures the dynamics of the replica of the secondary path. The state space representation of the system is then

$\overbrace{\left[\begin{array}{c}W(k+1) \\ \theta(k+1)\end{array}\right]}^{\xi_{(k+1)}}=\overbrace{\left[\begin{array}{lr}I_{(2 N+1) \times(2 N+1)} & 0 \\ B_{s}(k) h_{k}^{*} & A_{s}(k)\end{array}\right]}^{F_{k}}\left[\begin{array}{c}W(k) \\ \theta(k)\end{array}\right]$

where

$h_{k}=[x(k) r(k-1) \cdots r(k-N) r(k-1) \cdots r(k-N)]^{T}$

captures the effect of the reference input $x(\cdot)$. Note that

$r(k)=x(k) a_{0}(k)+r(k-1) b_{1}(k)+\cdots+r(k-N) b_{N}(k)$,

$r(-1)=\cdots=r(-N)=0$

and therefore, the system dynamics are nonlinear in the IIR filter parameters. For this system, the derived measured output is

$$
m(k)=\overbrace{\left[\begin{array}{ll}
D_{s}(k) h_{k}^{*} & C_{s}(k)
\end{array}\right]}^{H_{k}}\left[\begin{array}{c}
W(k) \\
\theta(k)
\end{array}\right]+\mathcal{V}_{m}(k)
$$

where $m(k)$ should be constructed at each step according to Equation (1). Once again, the measurement equation is also nonlinear in the parameters of the IIR filter. Now, define a generic linear combination of the states as the 
desired quantity to be estimated

$$
s(k)=\overbrace{\left[\begin{array}{ll}
L_{1, k} & L_{2, k}
\end{array}\right]}^{L_{k}}\left[\begin{array}{c}
W(k) \\
\theta(k)
\end{array}\right]
$$

Note that $m(\cdot) \in \mathcal{R}^{p \times 1}, s(\cdot) \in \mathcal{R}^{q \times 1}, \theta(\cdot) \in \mathcal{R}^{r \times 1}$, and $W(\cdot) \in \mathcal{R}^{(2 N+1) \times 1}$. All matrices are then of appropriate dimensions.

To allow for a simplified solution (see Section 3 ), of all choices available for $L_{K}, L_{K}=H_{k}$ is considered here. In principle, any estimation algorithm can now be used to generate $\hat{s}(k \mid k) \triangleq \mathcal{F}(m(0), \cdots, m(k)$ ) (a causal estimate of the desired quantity, $s(k))$ such that some closeness criterion is met. This paper focuses on an $H_{\infty}$ estimation criterion. Here, the main objective is to limit the worst case energy gain from the measurement disturbance and the initial condition uncertainty to the error in a causal estimate of $s(k)$. In other words, it is desired to find an $H_{\infty}$ suboptimal causal estimator $\hat{s}(k \mid k)=\mathcal{F}(m(0), \cdots, m(k))$ such that

$$
\sup _{\mathcal{V}_{m}, \xi_{0}} \frac{\sum_{k=0}^{M}[s(k)-\hat{s}(k \mid k)]^{*}[s(k)-\hat{s}(k \mid k)]}{\xi_{0}^{*} \Pi_{0}^{-1} \xi_{0}+\sum_{k=0}^{M} \mathcal{V}_{m}^{*}(k) \mathcal{V}_{m}(k)} \leq \gamma^{2}
$$

Note that, in this case

1. There is no statistical assumption regarding the measurement disturbance. Therefore, the error in the modeling of the primary path can be easily treated as a component of the measurement disturbance. For large modeling error, however, the performance can be expected to deteriorate.

2. A closed form solution to the nonlinear $H_{\infty}$ estimation problem is not available. To derive a recursion for the filter parameter update, we apply the following approximation: At each time step, replace the IIR filter parameters in Equation (3) with their best available estimate. This reduces the problem into a linear $H_{\infty}$ estimation problem for which a solution similar to that in [3] exists ${ }^{2}$.

\section{$3 H_{\infty}$-Optimal Solution}

In this section, we only quote the $\gamma$-suboptimal filtering solution for the linearized $H_{\infty}$-estimation problem $[5,8]$. The arguments for the optimal value of $\gamma$ for the solution to the linearized problem and the simplifications that follows are similar to those presented in [3] and hence are not repeated here.

\footnotetext{
${ }^{2}$ As Figure 3 suggests, feedback is an integral part of an IIR filter structure. The discussion in Section 2.1 showed that this feedback results in the nonlinearity in the system dynamics. The same holds true when a feedback path exists such that the reference signal is contaminated by the output of the adaptive filter itself (Figure 1). Our treatment of the nonlinearity in the case of an IIR filter, i.e. replacing the IIR filter parameters in Equation (3) with their best available estimates, carries over to the case where such a feedback path exists.
}

$3.1 \gamma$-Suboptimal Finite Horizon Filtering Solution

Theorem [5]: Consider the system in Figure 3 and described by Equations (2)-(5). Assume that the linearizing approximation discussed in the previous section is applied. A level- $\gamma H_{\infty}$ filter that achieves (6) exists if, and only if, the matrices $R_{k}$ and $R_{e, k}$ defined by

$$
R_{e, k}=\overbrace{\left[\begin{array}{rr}
I_{p} & 0 \\
0 & -\gamma^{2} I_{q}
\end{array}\right]}^{R_{k}}+\left[\begin{array}{c}
H_{k} \\
L_{k}
\end{array}\right] P_{k}\left[\begin{array}{ll}
H_{k}^{*} & L_{k}^{*}
\end{array}\right]
$$

have the same inertia for all $0 \leq k \leq M$, where $P_{0}=\Pi_{0}$ and $P_{k}>0$ satisfies the Riccati recursion

$$
P_{k+1}=F_{k} P_{k} F_{k}^{*}-K_{p, k} R_{e, k} K_{p, k}^{*}
$$

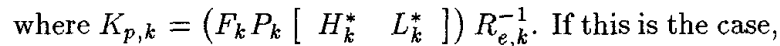
then the central $H_{\infty}$ estimator is given by

$$
\begin{gathered}
\hat{\xi}_{k+1}=F_{k} \hat{\xi}_{k}+K_{1, k}\left(m(k)-H_{k} \hat{\xi}_{k}\right), \quad \hat{\xi}_{0}=0 \\
\hat{s}(k \mid k)=L_{k} \hat{\xi}_{k}+\left(L_{k} P_{k} H_{k}^{*}\right) R_{H e, k}^{-1}\left(m(k)-H_{k} \hat{\xi}_{k}\right)
\end{gathered}
$$

with $K_{1, k}=\left(F_{k} P_{k} H_{k}^{*}\right) R_{H e, k}^{-1}$ and $R_{H e, k}=I_{p}+H_{k} P_{k} H_{k}^{*}$. The optimal value of $\gamma$ is 1 (see Refs. $[2,3]$ ), and for this optimal value the Riccati equation in (8) reduces to $P_{k+1}=F_{k} P_{k} F_{K}^{*}$ ).

\subsection{Important Remarks}

1. As in the case for the FIR filter design, the estimationbased approach to the design of the adaptive IIR filter requires the solution to only one Riccati equation. Furthermore, the Riccati solution propagates forward in time and does not involve any information regarding the future of the system or the reference signal. Thus, the resulting adaptive algorithm is real-time implementable.

2. As mentioned in Section 3.1, the Riccati update (for the simplified solution) reduces to a Lyapanov recursion which always generates a positive definite $P_{k}$ as long as $P_{0}>0$. This eliminates the need for computationally expensive checks for positive definiteness of $P_{k}$ at each step. 3. In general, the solution to an $H_{\infty}$ filtering problem requires verification of the fact that $R_{k}$ and $R_{\epsilon, k}$ are of the same inertia at each step. This can be a computationally expensive task. Our formulation of the problem eliminates the need for such checks by allowing a definitive answer to the feasibility of $\gamma=1$. This also guarantees that a breakdown in the solution will not happen.

4. With no need to verify the solutions at each step, the computational complexity of the estimation based approach is $O\left(n^{3}\right)$ (in calculating $F_{k} P_{k} F_{K}^{*}$ ), where $n=$ $2 N+1$ (number of IIR filter Parameters) $+n_{\text {sec-path }}$ (the order of the secondary path). The special structure of $F_{k}$ however reduces the computational complexity involved to $O\left(n_{s e c-p a t h}^{3}\right)$. This can be a substantial reduction in the computation when $2 N+1$ is large compared to $n_{\text {sec-path }}$.

\section{Estimation-Based Adaptive Algorithm}

In this section, we use three sets of variables to describe the adaptive algorithm, (i) $\hat{W}(k)$ and $\hat{\theta}(k)$ (best estimate 
of the state at time $k$ ), (ii) $\theta_{\text {actual }}, u(k) \triangleq h_{k}^{*} \hat{W}(k), y(k)$, and $d(k)$ (the actual value of the variables), and (iii) adaptive algorithm's internal copy of a variable (referred to with subscript "copy"). See [3] for a detailed description of these variables. We outline the implementation algorithm as follows:

1. Start with $\hat{W}(0)=\hat{W}_{0}, \hat{\theta}(0)=\hat{\theta}_{0}$ as the best initial guess for the state vector in the approximate model of the primary path. Assume that the IIR filter starts with $r(-1)=\cdots=r(-N)=0$. Also consider $\theta_{\text {actual }}(0)=\theta_{\text {actual }, 0}$, while the adaptive algorithm assumes that $\theta_{\text {copy }}(0)=\theta_{\text {copy }, 0}$. Furthermore, $d(0)$ is the initial output of the primary path. Then, for $0 \leq k \leq$ $M$ (finite horizon):

2. Form $h_{k}$ (with the available estimate of the parameter vector $\hat{W}(k))$,

3. Form the control signal $u(k)=h_{k}^{*} \hat{W}(k)$,

4. Apply the control signal to the secondary path. The actual output and the new state vector have dynamics

$$
\begin{aligned}
\theta_{\text {actual }}(k+1) & =A_{s}(k) \theta_{\text {actual }}(k)+B_{s}(k) u(k) \\
y(k) & =C_{s}(k) \theta_{\text {actual }}(k)+D_{s}(k) u(k)
\end{aligned}
$$

5. Propagate the internal copy of the state vector and the output of the secondary path as

$$
\begin{aligned}
\theta_{\text {copy }}(k+1) & =A_{s}(k) \theta_{\text {copy }}(k)+B_{s}(k) u(k) \\
y_{\text {copy }}(k) & =C_{s}(k) \theta_{\text {copy }}(k)+D_{s}(k) u(k)
\end{aligned}
$$

6. Form the derived measurement, $m(k)$, using the direct measurement $e(k)$ and the copy of the output of the secondary path $m(k)=e(k)+y_{\text {copy }}(k)$. Note that $e(k)$ is the error measured after the control signal $u(k)$ is applied.

7. Use the $H_{\infty}$-optirnal estimator's state update, Equations (9), to find the $H_{\infty}$-optimal estimate of the optimal IIR filter parameters in Figure 3 (i.e. $\hat{W}(k+1)$ ). Note that $\hat{\theta}(k+1)$ should also be stored for the next estimation update.

8. If $k \leq M$, go to 2 .

The example in the following section indicates that this algorithm performs well. We are currently investigating the impact of the use of $y_{c o p y}$ in this algorithm.

\section{Simulation}

This section examines the performance of the adaptive algorithm described in Section 4 . For the simulations in this section, second order systems, $P(z)=$ $\frac{z-0.3}{(z+0.4-j 0.8)(z+0.4+j 0.8)}$, and $S(z)=\frac{z-0.3}{(z+0.65-j 0.7)(z+0.65+j 0.7)}$, (with different damping ratios and resonance frequencies) are used as primary and secondary paths. The primary path is unknown to the adaptive algorithm. We use a second order adaptive IIR filter (and hence the parameter vector for the IIR filter is of length 5). We examine the performance of the adaptive IIR filter with and without feedback contamination. For the feedback path another second order system, $F(z)=\frac{z-0.3}{(z+0.2-j 0.75)(z+0.2+j 0.75)}$, is used. A multi-tone signal, $x(k)=\sum_{i=1}^{3} 10 \sin \left(2 \pi f_{i} k \Delta t\right)$ (where $f_{1}=3.0, f_{2}=4.5$, and $f_{3}=15 \mathrm{~Hz}$ ), is used as the reference signal. As measurement noise, $\mathcal{V}_{m}(k)$, we use a zero mean, normally distributed random variable with variance 0.01 . Furthermore, $\Delta t=0.01$ seconds and $P_{0}=0.05 I$

Figure 4 captures the performance of the adaptive IIR filter with the multi-tone reference signal described above when no feedback contamination exists. The error plot indicates an effective cancellation of the output of the primary path in roughly 1.5 seconds. Figure 5 clearly indicates that the existence of a feedback path from the output of the adaptive filter to the reference signal, results in a slower convergence of the adaptive IIR filter. Nevertheless, the adaptive filter successfully reduces the error in noise cancellation.

\section{Conclusion}

We have introduced a systematic, estimation-based approach to the design of adaptive IIR filters. This work extends our previous results in [3] (where an estimationbased approach to the design of FIR filters is introduced). We have also proposed an appropriate framework in which an IIR (as well as an FIR) filter can be designed when the reference signal is contaminated by a feedback from the output of the adaptive filter. The proposed algorithm uses an $H_{\infty}$ filtering solution to limit the worst case energy gain from the measurement disturbance and initial condition uncertainty to the residual error energy. We have shown that this worst case energy gain is unity, and have exploited the structure of the formulation to simplify the filtering solution.

In our view, the proposed formulation in this paper provides an appropriate framework for robustness studies of adaptive filters. Furthermore, systematic optimization of the filter parameters (such as the order of the IIR filter) may be investigated.

\section{Appendix A: Adaptive IIR Filter Design When Feedback Contamination Exists}

Figure 6 reflects the block diagram for an approximate model of the primary path when a feedback path from the output of the IIR filter to its input exists. The derivation of an adaptive IIR filter in this case paralles that in Sections 2 and 3 . Therefore, only the state space representation of this approximate model is outlined here:

$\left[\begin{array}{c}\overbrace{W(k+1)}^{\xi_{(k+1)}} \\ \theta(k+1) \\ \varphi(k+1)\end{array}\right]=\overbrace{\left[\begin{array}{lcr}I_{(2 N+1) \times(2 N+1)} & 0 & 0 \\ B_{s}(k) h_{k}^{*} & A_{s}(k) & 0 \\ B_{f}(k) h_{k}^{*} & 0 & A_{f}(k)\end{array}\right]}^{F_{k}}\left[\begin{array}{c}W(k) \\ \theta(k) \\ \varphi(k)\end{array}\right]$

where $\varphi(k)$ is the state vector for the feedback path, and $h_{k}$ and $W(k)$ are the same as in Section 2.1. Note 1.liat

$$
\begin{aligned}
x(k) & =r e f(k)+D_{f}(k) h^{*}(k) W(k)+C_{s}(k) \varphi(k), \\
r(k) & =a_{0} x(k)+b_{1} r(k-1)+\cdots+b_{N} r(k-N), \\
r(-1) & =\cdots=r(-N)=0
\end{aligned}
$$

where the contamination of the reference signal with the feedback from the output of the adaptive filter is evident. 
For this system the derived measured output, $m(k)$, is described as

$$
m(k)=\overbrace{\left[\begin{array}{lll}
D_{s}(k) h_{k}^{*} & C_{s}(k) & 0
\end{array}\right]}^{H_{k}}\left[\begin{array}{c}
W(k) \\
\theta(k) \\
\varphi(k)
\end{array}\right]+\mathcal{V}_{m}(k)
$$

while the quantity to be estimated, $s(k)$, is

$$
s(k)=\overbrace{\left[\begin{array}{lll}
D_{s}(k) h_{k}^{*} & C_{s}(k) & 0
\end{array}\right]}^{L_{k}}\left[\begin{array}{c}
W(k) \\
\theta(k) \\
\varphi(k)
\end{array}\right]
$$

Note that $H_{k}=L_{k}$. Obviously, system dynamics are nonlinear in IIR filter parameters. The disturbance attenuation criterion of Equation (6) can now be used to pose a nonlinear $H_{\infty}$-filtering problem. The approximate linear solution to this problem parallels that in Section 3 and is not repeated here. It is clear that the above mentioned formulation also applies when the adaptive IIR filter is replaced with an adaptive FIR filter.

\section{References}

1. B. Widrow and S.D. Stearns, Adaptive Signal Processing, Englewood Cliffs, NJ: Prentice-Hall, 1985.

2. B. Hassibi, A.H. Sayed and T. Kailath, " $H_{\infty}$ Optimality of the LMS Algorithm," IEEE Trans. on Signal Processing, Vol. 44, No. 2, pp. 267-280, February 1996.

3. B. Sayyarrodsari, J.P. How, B. Hassibi, and A. Carrier, "An $H_{\infty}$-Optimal Alternative to the FxLMS Algorithm", Submitted to American Control Conf. 1998.

4. S.M. Kuo and D.R. Morgan, Adaptive Noise Control Systems, Wiley Series in Telecommunication and Signal Processing, John Wiley \& Sons, Inc., 1996.

5. B. Hassibi, A.H. Sayed, and T. Kailath, "Indefinite Quadratic Estimation and Control", SIAM Studies in Applied Mathematics, 1998.

6. S.M. Kuo and J. Chen, "Multiple-Microphone Acoustic Echo Cancellation System With the Partial Adaptive Process", Digital Signal Process., No. 3, pp 54-63, Jan. 1993.

7. L.J. Eriksson, M.C. Allie, and R.A. Greiner, "The Selection and Application of an IIR Adaptive Filter for Use in Active Sound Attenuation", IEEE Trans, on Acous., Speech, Signal Processing, No. 35, pp. 433-437, April 1987.

8. P.P. Khargonekar, and K.M. Nagpal, "Filtering and Smoothing in an $H^{\infty}$-Setting," IEEE Trans. Automat. Control, vol. 36, pp. 151-166, 1991.
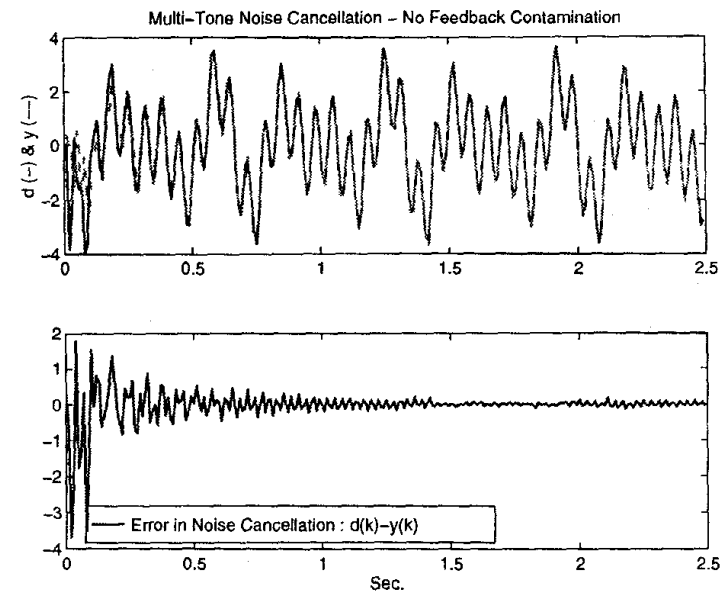

Fig. 4: The performance of the adaptive IIR filter in cancellation of the output of the secondary without feedback contamination
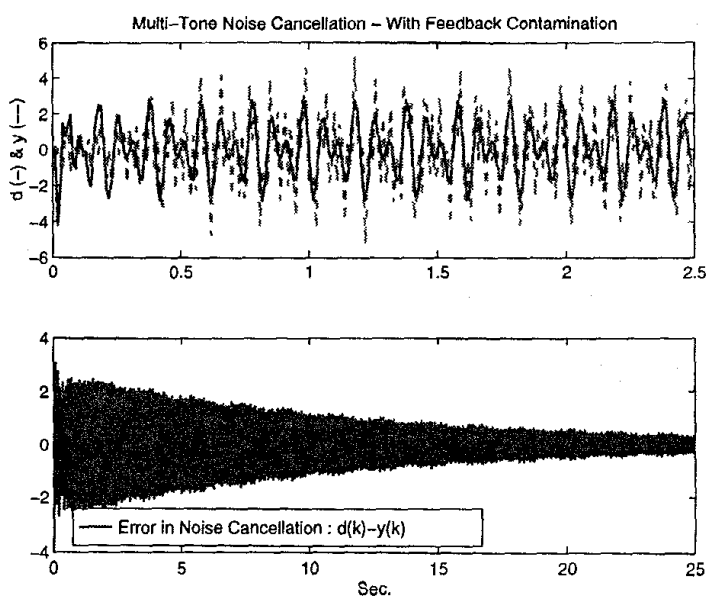

Fig. 5: The performance of the adaptive IIR filter when the reference signal in the presence of feedback contamination. Note that for clarity, $y(k)$ and $d(k)$ are plotted for only 2.5 seconds.

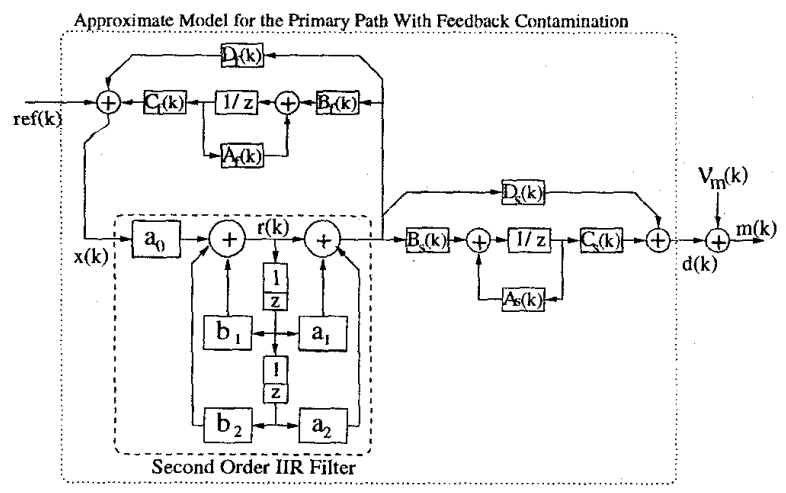

Fig. 6: Block diagram of the approximate model for the primary path in the presence of the feedback path 